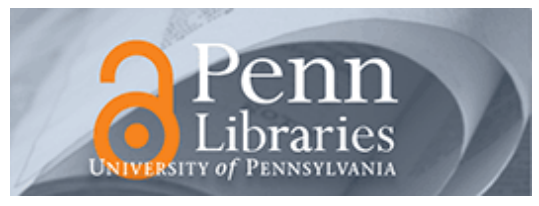

University of Pennsylvania ScholarlyCommons

3-13-2009

\title{
Transporting an Image through a Subwavelength Hole
}

\author{
Mario G. Silveirinha
}

University of Coimbra

Nader Engheta

University of Pennsylvania, engheta@ee.upenn.edu

Follow this and additional works at: https://repository.upenn.edu/ese_papers

\section{Recommended Citation}

Mario G. Silveirinha and Nader Engheta, "Transporting an Image through a Subwavelength Hole", . March 2009.

Transporting an Image through a Subwavelength Hole Mario G. Silveirinha and Nader Engheta, Phys. Rev. Lett. 102, 103902 (2009), DOI:10.1103/PhysRevLett.102.103902

Copyright American Physical Society. Reprinted from Physical Review Letters, Volume 102, Article 103902, March 2009.

Publisher URL: http://link.aps.org/doi/10.1103/PhysRevLett.102.103902

This paper is posted at ScholarlyCommons. https://repository.upenn.edu/ese_papers/483

For more information, please contact repository@pobox.upenn.edu. 


\title{
Transporting an Image through a Subwavelength Hole
}

\author{
Abstract \\ The manipulation of optical waves in the subwavelength scale is limited by diffraction. In the vicinity of a \\ narrow aperture, the amplitude of the electric field is approximately uniform and the transmissivity is \\ extremely low. Here we show that despite these fundamental constraints it may be possible to transport \\ and redirect a complex electromagnetic image through a tiny subwavelength hole with diameter \\ considerably smaller than the diameter of the image, without losing the subwavelength details. The \\ proposed concepts hold promise for an unprecedented manipulation of the electromagnetic and optical \\ fields in the nanoscale with potential applications in imaging and sensing.

\section{Comments} \\ Transporting an Image through a Subwavelength Hole Mario G. Silveirinha and Nader Engheta, Phys. Rev. \\ Lett. 102, 103902 (2009), DOI:10.1103/PhysRevLett.102.103902 \\ Copyright American Physical Society. Reprinted from Physical Review Letters, Volume 102, Article \\ 103902, March 2009. \\ Publisher URL: http://link.aps.org/doi/10.1103/PhysRevLett.102.103902
}




\title{
Transporting an Image through a Subwavelength Hole
}

\author{
Mário G. Silveirinha ${ }^{1,2, \dagger}$ and Nader Engheta ${ }^{1, *}$ \\ ${ }^{1}$ University of Pennsylvania, Department of Electrical and Systems Engineering, Philadelphia, Pennsylvania, USA \\ ${ }^{2}$ University of Coimbra, Department of Electrical Engineering-Instituto de Telecomunicações, Portugal \\ (Received 26 October 2008; revised manuscript received 25 January 2009; published 13 March 2009)
}

\begin{abstract}
The manipulation of optical waves in the subwavelength scale is limited by diffraction. In the vicinity of a narrow aperture, the amplitude of the electric field is approximately uniform and the transmissivity is extremely low. Here we show that despite these fundamental constraints it may be possible to transport and redirect a complex electromagnetic image through a tiny subwavelength hole with diameter considerably smaller than the diameter of the image, without losing the subwavelength details. The proposed concepts hold promise for an unprecedented manipulation of the electromagnetic and optical fields in the nanoscale with potential applications in imaging and sensing.
\end{abstract}

PACS numbers: 42.70.Qs, 42.30.Va, 78.66.Sq

In the past decade, there has been a great interest in the development of new techniques that may allow overcoming the effects of diffraction using metamaterials and plasmonic phenomena [1]. Up to this date, most of the proposals rely on the excitation of surface plasmon polaritons: strongly localized waves that emerge at interfaces between a metal and dielectric material, and that due to their characteristic short wavelengths may enable several interesting phenomena to occur, such as extraordinary optical transmission [2]. It was reported in Ref. [2] that due to the excitation of surface plasmon polaritons the transmission efficiency of arrays of very subwavelength holes in a metallic film may be orders of magnitude greater than predicted by conventional aperture theory.

In this Letter, we propose a completely different paradigm to propagate electromagnetic energy through a subwavelength aperture. While the phenomenon of enhanced transmission may allow optical energy to go through a tiny hole [2], here we show that a complex optical image may be squeezed and bent through the hole without losing its subwavelength fine details. The idea is to first sample the image "pixel by pixel" using an array of parallel nanowires, and then, in a second step, to "squeeze" the electric current associated with each pixel through the tiny hole, with essentially no reflection. This process can be understood by examining Fig. 1, which reports a possible geometry for the proposed imaging device. The system consists of 217 nanowires that uniformly sample the image created by a given source, and are rerouted from the front interface to the back interface through a tiny circular hole in an opaque metallic screen with dimensions $W_{s} \times W_{s}$, such that $W_{s}=\lambda_{0} / 2$, where $\lambda_{0}=10.31 \mu \mathrm{m}$ is the design wavelength. The subwavelength hole has diameter $D_{h}=$ $W_{s} / 2.5=\lambda_{o} / 5=2 \mu \mathrm{m}$, which implies that the sampled image is compressed by a factor of $2.5 \times 2.5=6.25$ as it goes through the narrow aperture, followed by an expansion by the same factor after it exits the hole. The nanowires are assumed to be made of aluminum (Al) and have radius $R=34 \mathrm{~nm}$. For simplicity, it is assumed here that the straight parts of the nanowires with length $L_{w}=$ $2.58 \mu \mathrm{m}$ stand in air. The distance between adjacent nanowires in the air region is about $a \approx 286 \mathrm{~nm}$, and is reduced by a factor of 2.5 as the wires go through the tiny hole.

The idea of sampling the image is motivated by recent theoretical and experimental studies that demonstrated that arrays of metallic wires may be used to manipulate the near field in the subwavelength spatial scale [3]. However, here the main challenge is to guarantee that even though the metallic wires are bent and highly compacted in the vicinity of the tiny hole, it is possible to squeeze the energy associated with each individual pixel to the other side of the opaque screen, with essentially no reflection. The strategy that we propose to overcome this difficulty is inspired by the tunneling effect we discovered in [4] and by our work on optical nanocircuits [5] and requires that the opaque screen and part of the wires are embedded in a block of a low permittivity material, which is assumed here to be silicon carbide $(\mathrm{SiC})$ [the yellow (light gray) region in Fig. 1]. Following the experimental results of [6], the permittivity of $\mathrm{SiC}$ is such that $\varepsilon \approx 0.1 i$ at $\lambda_{0}=$ $10.31 \mu \mathrm{m}$, i.e., $\mathrm{SiC}$ behaves as a material with permittivity $\varepsilon$ near zero $(\mathrm{ENZ})$, with $\operatorname{Re}\{\varepsilon\} \approx 0$.

The electromagnetic response of the proposed "sampling, squeezing, and bending" device was characterized using a commercial full-wave electromagnetic simulator [7]. For simplicity, it was assumed in the simulations that the source is a nanoantenna shaped like the letter S. A time snapshot of the (normal component) electric field distribution created by the antenna at the front interface is depicted in panel (a) of Fig. 2. The corresponding images at the back interface (after transporting through the tiny hole) are reported in panels (b)-(c) for different wavelengths (other images and field animations at $\lambda=10.31 \mu \mathrm{m}$ are reported in the EPAPS supplementary material [8]). It is seen that, notwithstanding the very subwavelength dimensions of the hole and despite the complexity of the image, the field distributions at the output may closely mimic the image at the front interface. Indeed, it is quite remarkable how, 

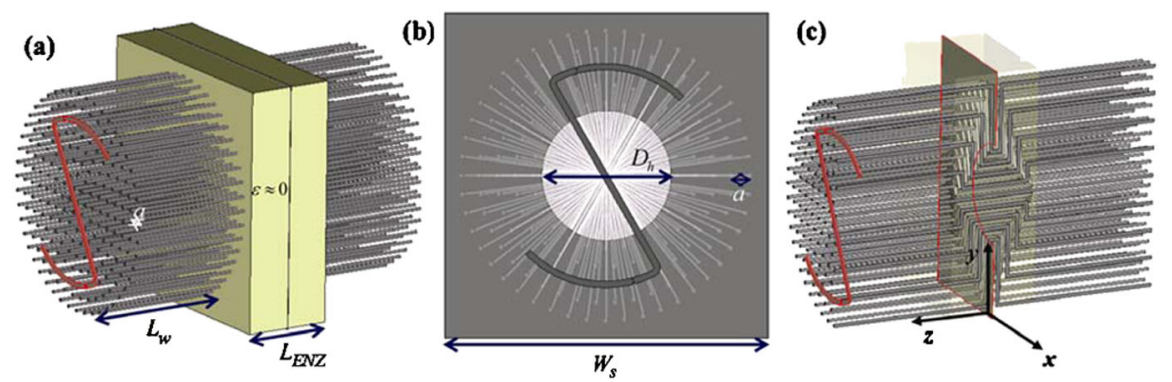

FIG. 1 (color online). Transporting an optical image through a tiny subwavelength hole: A bundle of metallic (Al) nanowires is redirected through an opaque metallic perfect electric conductor screen with a tiny subwavelength hole. The metallic screen is embedded in an ENZ material, silicon carbide (SiC). The thickness of the ENZ block is $L_{\mathrm{ENZ}}=0.2 \lambda_{0}=2 \mu \mathrm{m}$, where $\lambda_{0}=$ $10.31 \mu \mathrm{m}$ is the wavelength of operation. An S-shaped nanoantenna is placed close to the front interface. The screen has dimensions $W_{s} \times W_{s}$, with $W_{s}=5.2 \mu \mathrm{m}$. The subwavelength hole has diameter $D_{h}=2 \mu \mathrm{m}$. (a) Perspective view. (b) Front view (the wires go through the narrow hole from one side to the other). (c) Cut along the midplane normal to the $x$ direction.

despite the several bends suffered by the nanowires and despite the nanowires being so closely packed in the vicinity of the tiny hole, the subwavelength details of the original image may be reproduced at the back interface. In particular, when the permittivity of $\mathrm{SiC}$ (see the labels of Fig. 2) is near zero, the image at the front interface is transported and reproduced with little distortion at the output [8]. The bandwidth of the imaging may be estimated to be about $1 \%$ of the operating frequency, and is mainly limited by the frequency dispersion of the permittivity of $\mathrm{SiC}$, which varies relatively fast due to the proximity of a resonance at $\lambda=12.6 \mu \mathrm{m}$ [6]. The distance between the front and back interfaces is as large as $0.7 \lambda_{0}$. The resolution of the system is estimated to be roughly $2 a=\lambda_{0} / 18$. These results illustrate how the proposed "sampling and squeezing" concept may allow transporting, compressing, and expanding electromagnetic energy in the nanoscale and, in this way, enable image formation through a very narrow aperture with subwavelength resolution.

In order to explain the role of the ENZ material, we note that each nanowire may be regarded as a "sensor" that maps the sampled electric field into an electric current. Obviously, the electric current distribution along a given wire may be strongly perturbed by deformations and bends (which may completely modify the density of electric charge on the surface of the wire). Hence, in general one would expect that the electric current in a given nanowire at the back interface might be extremely small, even if the current injected at the front interface were significant. However, when the nanowires are embedded in a material with $\varepsilon \approx 0$, the situation changes drastically. In fact, consider a metallic wire embedded in a material with $\varepsilon=0$. (For simplicity in this discussion the wire is assumed to be a perfect electric conductor.) The geometry of the wire may be completely arbitrary, with an arbitrary number of bends, and possibly with a nonuniform cross section. The density of the surface (conduction) current is denoted by $\mathbf{J}_{c}$, and density of surface charge by $\sigma_{c}=\varepsilon E_{n}$, where $E_{n}$ is the normal component of the electric field. The current continuity requires that $\operatorname{div} \mathbf{J}_{c}=i \omega \sigma_{c}$, where div represents the surface divergence. Thus, in the $\varepsilon=0$ limit, if $E_{n}$ remains finite in the ENZ material, the density of the surface charge must vanish, $\sigma_{c}=0$, and hence $\operatorname{div} \mathbf{J}_{c}=$ 0 . This has a very important consequence: the flux of $\mathbf{J}_{c}$ is invariant through an arbitrary closed contour on the surface of the wire, or in other words the total current that crosses an arbitrary cross section of the wire is invariant. In particular, in the $\varepsilon=0$ limit the current injected in the ENZ material by a given nanowire at the front interface is exactly the same as the electric current that emerges at
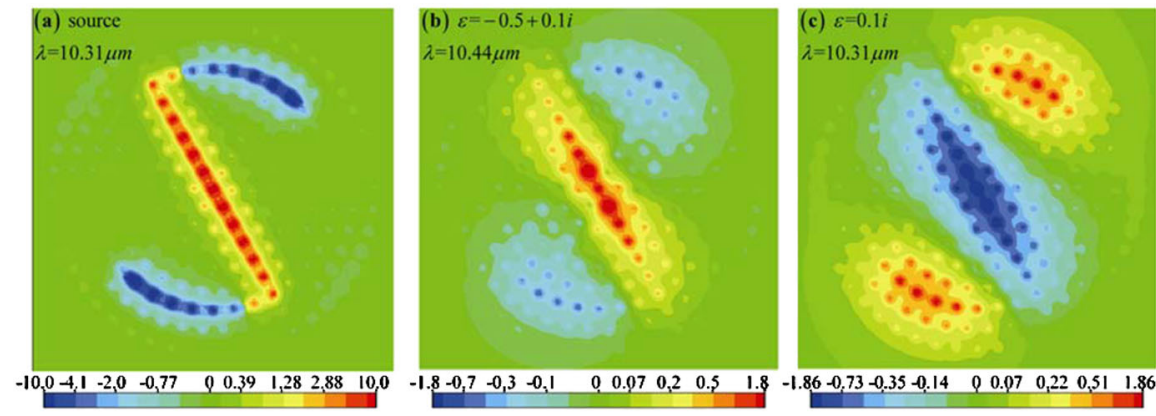

FIG. 2 (color online). Snapshot $(t=0)$ of the $E_{z}$ field component at the front interface [panel (a)] and at the back interface for different wavelengths [panels (b)-(c)], for the geometry of Fig. 1. The electric field is normalized to arbitrary unities. The permittivity of $\mathrm{SiC}$ at the considered wavelengths [6] is indicated explicitly in each panel. The best imaging occurs at $\lambda_{0}=10.31 \mu \mathrm{m}$ [panel (c)] when $\operatorname{Re}\{\varepsilon\}=0$, where the field distribution at the back interface is $180^{\circ}$ out of phase with respect to the front interface. 
the back interface, independent of the number of bends and the sharpness of the bends, of the total length of the nanowire, or of the distance between different nanowires. In this sense, the ENZ material can be regarded as an insulator for the conduction current in close analogy with the results reported in [5], where we suggested that ENZ materials may behave as insulators for the displacement current in an optical nanocircuit. Such a characteristic ensures that the environment around the tiny hole does not affect in any manner the current injected in the wires, preventing any form of mutual coupling between the currents associated with the different pixels of the image.

Clearly, in order that the image can be transported through the tiny hole, the current injected in the ENZ material (associated with a given "pixel") needs to be meaningful. This is ensured by the fact that the metallic rods are tightly packed in the ENZ region. Indeed, any two neighboring metallic nanowires may be regarded as a nanotransmission line, whose propagation characteristics may be described in terms of a characteristic inductance $L$ and capacitance $C$ per unit of length. The characteristic line impedance of a transmission line formed by two parallel perfect electric conductor wires with radius $R$ and separated by a distance $d$ is $Z_{0}=\sqrt{\frac{L}{C}}=\eta_{0} \sqrt{\frac{\varepsilon_{0}}{\varepsilon}} \frac{\varepsilon}{C}$, where $C=2 \pi \varepsilon / \cosh ^{-1}\left(\frac{d^{2}}{2 R^{2}}-1\right)$ is the per unit length capacitance and $\eta_{0}$ is the wave impedance in free space. Hence, for a fixed radius $R$, the line impedance decreases as the distance $d$ between the wires decreases, and increases as the permittivity of the background material $\varepsilon$ decreases. Thus, the line impedance may be kept invariant by reducing both the distance between the nanowires and $\varepsilon$. Hence, an ENZ material may be used to "match" nanotransmission lines with very different physical dimensions, as in the configuration of Fig. 1, and this justifies why the energy can be tunneled through the tiny hole. This effect is closely related to the tunneling phenomenon reported in [4], which was recently experimentally demonstrated at microwaves $[9,10]$. It may be proven that the current injected in the ENZ material is maximal when the length $L_{w}$ of the sections of the nanowires that stand in air (Fig. 1) is such that $L_{w}=0.25 m \lambda$, with $m=1,3,5, \ldots$.

An exciting property of the proposed imaging device is that it may not introduce a significant phase distortion in the transported image. Indeed, an inspection of Fig. 2 and of the field animations [8] reveals that the phase difference between the images at the front and back interfaces is nearly uniform and equal to $180^{\circ}$. This is quite remarkable given that the parts of the image associated with nanowires in the periphery of the wire bundle travel a longer distance than the parts associated with nanowires in the center, and this typically would imply that the pixels in the periphery should suffer a larger phase shift than the pixels in the center. The weak phase distortion clearly stems from the staticlike behavior of ENZ materials, which implies a slow phase variation in these media. Thus, independent of the length of the nanowire inside the ENZ, the phase shift suffered by every pixel is approximately $2 \pi \times 2 L_{w} / \lambda_{0}$, which for $L_{w}=0.25 \lambda_{0}$ yields $180^{\circ}$, consistent with the results reported in Fig. 2.

The fabrication of a device with the geometry shown in Fig. 1 may be technologically challenging. However, the structure can be considerably simplified if the image is compressed along a single direction of space. Quite interestingly, this simplified setup may even be feasible for the demonstration of this phenomenon at microwave frequencies, where the fabrication of the proposed structures may be easier. Even though in principle there are no solid-state ENZ materials readily available at microwave frequencies, their response may be effectively emulated using a microwave waveguide operated at cutoff, as shown in [9]. The proposed structure is represented in Fig. 3(a), and consists of two standard (closed) rectangular metallic waveguides with dimensions $W_{E} \times W_{H}=56 \mathrm{~mm} \times 102 \mathrm{~mm}$ filled with Teflon $(\varepsilon=2.0)$ and connected at right angles by ultranarrow channels with length $L_{\mathrm{ENZ}}$ (the metallic walls
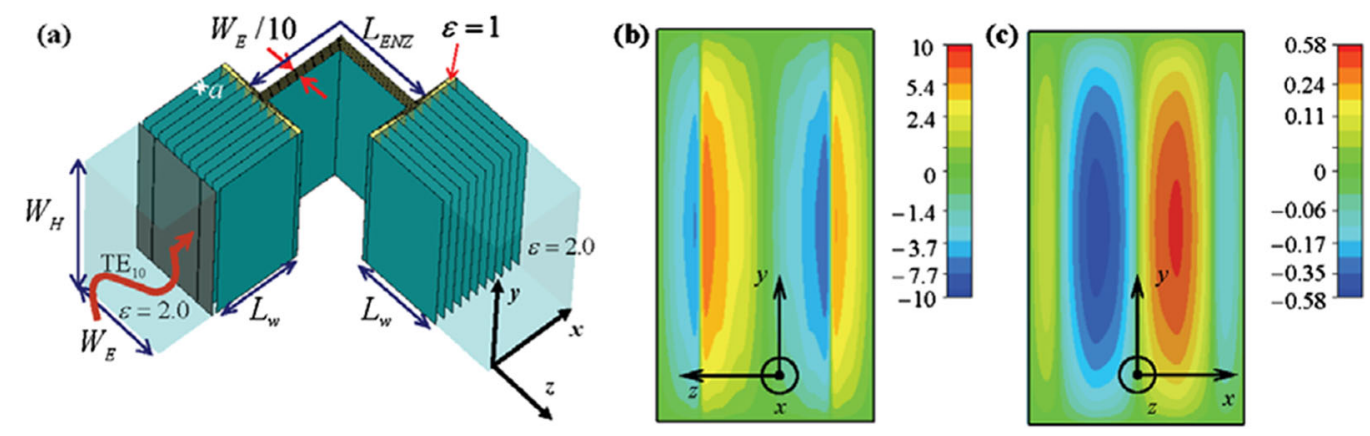

FIG. 3 (color online). Microwave design for the "sampling and squeezing" phenomenon: Panel (a): Two microwave rectangular metallic waveguides filled with Teflon $(\varepsilon=2.0)$ are connected at right angles by an array of ultranarrow channels filled with air $(\varepsilon=1.0)$. The $H$-plane width, $W_{H}$, is tuned so that at the frequency of operation $(1.47 \mathrm{GHz})$ the regions filled with Teflon behave effectively as a continuous material with $\varepsilon_{\text {eff,Teflon }}=1.0$, whereas the narrow channels filled with air behave as a material with $\varepsilon_{\text {eff,air }}=0$. The waveguide walls are assumed to be made of copper with conductivity $\sigma=5.8 \times 10^{7} \mathrm{~S} / \mathrm{m}$. The image is created by an opaque screen with two narrow slits illuminated by the fundamental TE 10 mode. Panel (b): Snapshot $(t=0)$ of the electric field normal component (normalized to arbitrary unities) at the input plane. Panel (c): Similar to panel (b) but at the output plane. 
enclosing the structure are not shown in Fig. 3 to allow us to view the internal structure of the setup). The waveguide is filled with an array of metallic parallel plates whose role is to sample the electric field, and guide the sampled signals through the ultranarrow channels, providing a 10fold image compression. The $H$-plane width (i.e., the width in the $y$ direction), $W_{H}=102 \mathrm{~mm}$, is chosen so that the narrow channels operate at their cutoff frequency, and thus effectively emulate the response of an ENZ material [9]. Indeed, the propagation of the fundamental $\mathrm{TE}_{10}$ mode in a rectangular metallic waveguide is in many ways analogous to the propagation of a transverse electromagnetic plane wave in a continuous medium characterized by the effective (relative) permittivity [9], $\varepsilon_{\text {eff }}=\varepsilon_{d}-\left(\frac{\pi c}{W_{H} \omega}\right)^{2}$, where $c$ is the speed of light in vacuum, $\omega$ is the angular frequency, and $\varepsilon_{d}$ is the (relative) permittivity of the material that fills the waveguide. The narrow channels in Fig. 3(a) are filled with air $\left(\varepsilon_{d}=1\right)$, and thus their effective permittivity vanishes, $\varepsilon_{\text {eff,air }}=0$, at the cutoff frequency $f_{p}=$ 1.47 GHz. On the other hand, the wide waveguide regions are filled with Teflon $\left(\varepsilon_{d}=2.0\right)$ and thus have effective permittivity $\varepsilon_{\text {eff,Teflon }}=1.0$ at the same frequency. Notice that the effective permittivities of both the air and Teflon regions depend on frequency. These simple but very powerful analogies may enable the demonstration of the "sampling and squeezing" concept at microwaves, as shown next with full-wave simulations.

The image is produced by an opaque screen with two thin slits (width $0.5 \mathrm{~mm}$ ) oriented along the $y$ direction. The opaque screen is illuminated by the fundamental $\mathrm{TE}_{10}$ waveguide mode. This creates a very sharp electric field distribution at the input of the "sampling and squeezing" device, as illustrated in Fig. 3(b). The length of the metallic plates in the Teflon regions is tuned so that $L_{w}=$ $0.25 \lambda_{0} / \sqrt{\varepsilon_{\text {eff,Teflon }}}=51 \mathrm{~mm}$, similar to the first example in this Letter. The distance between the metallic plates in the Teflon region is $a=W_{E} / 10=5.6 \mathrm{~mm}$, and this value is reduced 10 times in the narrow channels. The total length of the central narrow channel is $L_{\mathrm{ENZ}}=102 \mathrm{~mm}$. The distance between the two slits at the input plane is $33.6 \mathrm{~mm}$, whereas the wavelength of the $\mathrm{TE}_{10}$ mode at the design frequency $f_{p}=1.47 \mathrm{GHz}$ is $\lambda_{\mathrm{TE} 10}=$ $\lambda_{0} / \sqrt{\varepsilon_{\text {eff,Teflon }}}=204 \mathrm{~mm}$.

The image at the output plane is depicted in Fig. 3(c), and the corresponding field animation is reported in [8]. It is seen that notwithstanding the fact that the narrow channels are operated at cutoff and that the image is compressed severalfold and the channels are bent, the electromagnetic fields and the image tunnel from the input plane to the output plane. Moreover, even though the propagation distance is a significant fraction of the wavelength and the distance between the slits is as small as $\lambda_{\mathrm{TE} 10} / 6$, the two slits are clearly resolved by the proposed device. By comparing the field animations at the input and output planes [8], it is clear that the image at the output is $180^{\circ}$ out of phase with respect to the image at the input. This gives further proof that the novel "sampling, squeezing, and bending" technique may not introduce significant phase distortion between different parts of the image, and may enable a unique control of the electromagnetic fields and images in the subwavelength scale.

In conclusion, we have introduced a novel concept to manipulate electromagnetic images in the nanoscale that may enable squeezing and bending a very complex image through a tiny, deeply subwavelength hole without losing the subwavelength details. This may be achieved by sampling the image, and by squeezing the electric current associated with each pixel through the subwavelength hole. ENZ materials play a central role in this technique due to their anomalous insulating properties for the conduction electric current, allowing the current in the wires to be spatially invariant while in the ENZ region, and because they may enable adapting nanotransmission lines with a large height mismatch. We proposed a design for the demonstration of these mechanisms at microwaves using a metallic waveguide operated at cutoff to emulate the response of the ENZ materials. We envision that the described ideas may have important applications in emerging nanotechnologies.

This work is supported in part by the U.S. Office of Naval Research (ONR) Grant No. N 00014 -07-1-0622, and by Fundação para a Ciência e Tecnologia, Grant. No. PDTC/EEA-TEL/71819/2006.

*To whom correspondence should be addressed: engheta@ee.upenn.edu ${ }^{\dagger}$ mario.silveirinha@co.it.pt

[1] J. B. Pendry, Phys. Rev. Lett. 85, 3966 (2000); N. Fang, H. Lee, C. Sun, and X. Zhang, Science 308, 534 (2005).

[2] T. W. Ebbesen, H. J. Lezec, H. F. Ghaemi, T. Thio, and P. A. Wolff, Nature (London) 391, 667 (1998).

[3] P. A. Belov, Y. Hao, and S. Sudhakaran, Phys. Rev. B 73, 033108 (2006); G. Shvets, S. Trendafilov, J. B. Pendry, and A. Sarychev, Phys. Rev. Lett. 99, 053903 (2007); P. Ikonen, C. Simovski, S. Tretyakov, P. Belov, and Y. Hao, Appl. Phys. Lett. 91, 104102 (2007).

[4] M. G. Silveirinha and N. Engheta, Phys. Rev. Lett. 97, 157403 (2006).

[5] N. Engheta, Science 317, 1698 (2007).

[6] W. G. Spitzer, D. Kleinman, and D. Walsh, Phys. Rev. 113, 127 (1959).

[7] CST Microwave Studio ${ }^{\mathrm{TM}}$ 2006B, CST of America, Inc., www.cst.com.

[8] See EPAPS Document No. E-PRLTAO-102-060912 for field animations and electric field images. For more information on EPAPS, see http://www.aip.org/pubservs/ epaps.html.

[9] B. Edwards, A. Alù, M. E. Young, M. Silveirinha, and N. Engheta, Phys. Rev. Lett. 100, 033903 (2008).

[10] R. Liu, Q. Cheng, T. Hand, J. J. Mock, T. J. Cui, S. A. Cummer, and D. R. Smith, Phys. Rev. Lett. 100, 023903 (2008). 